Supporting Information

\title{
Fabrication of Electronic Junctions between Oriented Multilayers of Photosystem I and the Electrodes of Optoelectronic Solid-State Devices
}

\author{
Hani Barhum, Chanoch Carmeli and Itai Carmeli ${ }^{*}$
}

H. Barhum, Prof. C. Carmeli, Departments of Biochemistry and Molecular Biology, Tel Aviv University, Tel Aviv 69978, Israel, ccarmeli@post.tau.ac.il

I. Carmeli, Department of Engineering and Institute for nano technology, Bar Ilan University, Ramat Gan 5290002, Israel.

\section{Experimental Section S}

Unique cysteine mutants were induced by site-directed mutagenesis in the $p s a B$ and $p s a C$ genes of photosystem I (PSI) from Synechocystis sp. PCC 6803 resulting in D235C/Y634C and D31C, respectively (Figure S1) as earlier described by us. ${ }^{1}$ PSI was isolated from thylakoid membranes by solubilizing with n-dodecyl $\beta$-D-maltoside ( $\beta$-DM) and purification on DEAEcellulose columns and on a sucrose gradient. The isolation of PSI, the analysis chlorophyll content and photochemical activity determined by flash-induced transient oxidation of P700 at $\Delta \mathrm{A} 820$ and at $\Delta \mathrm{A} 700 \mathrm{~nm}$ were as described. ${ }^{2}$ In both the cysteine mutant and the native PSI, a half-time of 25 ms for the reduction of P700 was recorded.

Monolayers PSI were fabricated on glass slides covered with 200nm gold over $10 \mathrm{~nm}$ titanium and pretreated with dithio-bis-maleimidoethane (DTME) in ethanol. The PSI mutants were incubated in reaction mixture containing $\sim 0.5 \mathrm{mg}$ chlorophyll $/ \mathrm{ml}, 0.1 \mathrm{M} \mathrm{K}, \mathrm{NaH}_{2} \mathrm{PO}_{4}, \mathrm{pH} 6.8$; $\mathrm{NaCl} 0.15 \mathrm{M}$ and $0.05 \% \beta$-DM, for 20 min at $20^{\circ} \mathrm{C}$ than washed with distilled water. Alternatively, PSI cysteine mutants were self-assembled directly to gold surfaces as earlier described. ${ }^{1}$ Multilayer 
were fabricated layer by layer, as previously describe by $\mathrm{us}^{3}$ by incubation of PSI monolayer in a medium containing 2 mM m-Maleimidobenzoyl-N-hydroxysulfosuccinimide ester (S-MBS), with the same buffer at $\mathrm{pH} 7.2$ for $1 \mathrm{~h}$ at $20^{\circ} \mathrm{C}$ than wash of unreacted reagent. The modified PSI layer was incubation with PSI mutants in the same buffer at pH 6.8 for 30 min than washed in distilled water to remove unbound proteins. When indicated the modification of the monolayer by S-MBS was preceded by binding of $p$-phenylenediamine (PPD) to free carboxyl residues of PSI layers using cabodiimid chemistry as earlier described ${ }^{4}$. The monolayer was incubated in a solution containing 2 mM 1-Ethyl-3-[3-dimethylaminopropyl]carbodiimide hydrochloride (EDC), 5 mM sulfo-N-hydroxysulfosuccinimide (S-NHS), 0.1M 2-(N-morpholino)ethanesulfonic acid (MES), $\mathrm{pH}$ 6; $\mathrm{NaCl} 0.15$ for $20 \mathrm{~min}$ at $20^{\circ} \mathrm{C}$ than washed with the same buffer. The modified PSI monolayer were incubated in a reaction mixture containing: $5 \mathrm{mM} \mathrm{PPD}, 0.1 \mathrm{M} \mathrm{K}, \mathrm{NaH}_{2} \mathrm{PO}_{4}, \mathrm{pH}$ 7.2; $\mathrm{NaCl} 0.15 \mathrm{M}, 10 \% \mathrm{DMSO}$ for $2 \mathrm{~h}$ at $20^{\circ} \mathrm{C}$. The multilayers were incubated in a reaction containing: $0.13 \mathrm{mM}$ (1,2-Methanofullerene C60)-61-carboxylic acid (CF), $0.1 \mathrm{M}$, Tricine $\mathrm{pH}$ 7.5;10\% DMSO for $2 \mathrm{~h}$ than the excess of fullerene washed with the same buffer.

Contact potential difference (CPD) was determined by KPFM in a 'lift mode' in an AFM model NTMDT, equipped with a custom-made 1300-nm wavelength feedback laser. The CPD is extracted in the conventional way by nullifying the output signal of a lock-in amplifier, which measures the electrostatic force at the first resonance frequency ${ }^{5}$. AFM topography and KPFM were recorded in sequential scans at a scan rate of $1 \mathrm{~Hz} ; 512$ lines. Samples were illuminated by diode laser $670 \mathrm{~nm} 40 \mathrm{mV}$ maximum power. The absorption of PSI multilayers was measured by Cary 5000 spectrometer equipped with total absorption attachment (Figure S7). Ellipsometry was determined by a Woollam Alpha-SE (Figure S8).

Photolithography: The electrodes were defined by photolithography on glass surfaces. Glass wafers surfaces were cleaned by sonication in isopropanol acetone solution. The clean surface was spin coated by a primer hexamethyldisilazane (HMDS) and then spin coated by a photoresist AZ-15/18 that was soft-baked at $110{ }^{\circ} \mathrm{C}$ (see review by Truskett, and Watts. ${ }^{6}$ ). After prebaking, the photoresist was exposed to a pattern of intense UV light through chromium coated Kapton mask in MJB3 Mask Aligner apparatus. Az-726 developer was used to develop the exposed photoresist. Distilled water were used to end the development process. The resulting wafer was then hard-baked at $120^{\circ} \mathrm{C}$ and incubated in $\mathrm{HCl}, \mathrm{H}_{2} \mathrm{SO}_{4}$ solution. Bottom gold electrodes were deposited by the use of chemical vapor deposition. A lift-off procedure was performed by 
immersing the wafer in acetone for $15 \mathrm{~min}$ at room temperature and finally washed in acetone isopropanol and ethanol solution and dried under nitrogen. Glass coated ITO wafers were used to define ITO electrodes. The Kepton masked were designed for UV transparency in the space between the defined electrodes. Following UV exposure the photoresist was developed and the exposed ITO was etched by acid. The etching was followed by lift-off of the residual photoresist as described above. The Kepton masks were also used for chemical vapor deposition evaporate the top gold electrodes and for spin-coat conducting polymer.

The image for the shadow masks originated from a computerized data file using clew program. This data file was converted to a series of polygons and written onto a square of Kapton paper covered with a layer of chromium using a photolithographic process. A 342nm UV laser beam is used to expose the pattern over the surface of the substrate. The Kepton masks were used chemical vapor deposition evaporate the top gold electrodes, spin-coat conducting polymer and etching of ITO on glass wafers.

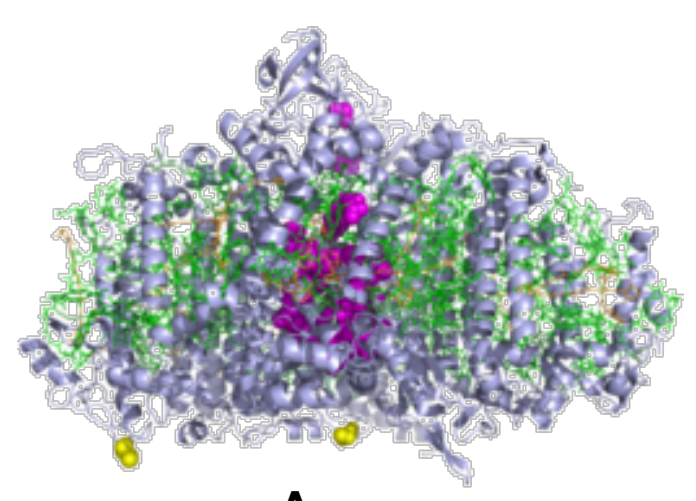

A

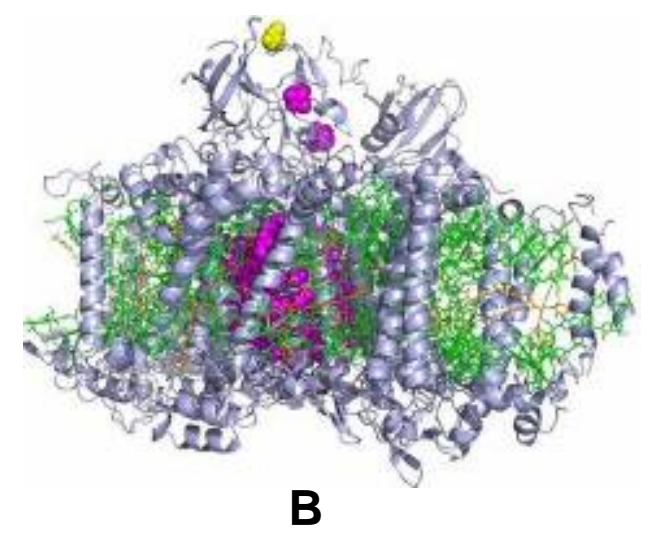

Figure S1. The structure of PSI mutants. The molecular structures of PSI mutants D235C/Y634C (A) and D31C (B) from Synechocystis sp. PCC 6803. In the structure polypeptide chains (gray ribbons), the chlorophylls (green rods), the electron transfer chain (magenta space fill) and the cysteine mutations (yellow space fill). The images of PSI were constructed from $\mathrm{x}$ ray crystallographic data published by ${ }^{7}$ PBD $1 \mathrm{JB} 0$. 

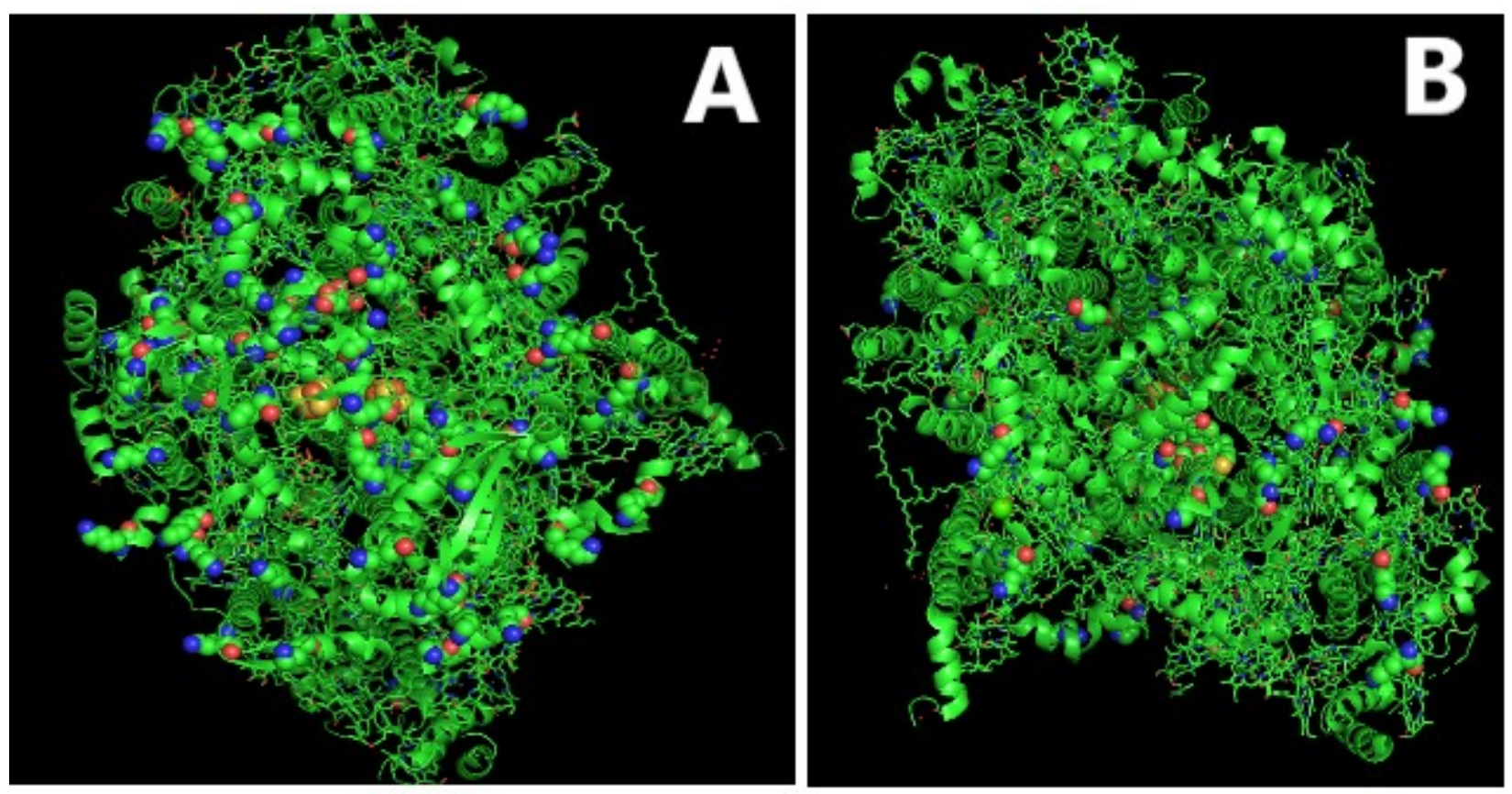

Figure S2. Exposed amines of lysine residues located on the surfaces of PSI. Multiple exposed amines of the lysine residues (blue spheres) are shown on the donor (A) and the acceptor surfaces of PSI. These amines can react with m-Maleimidobenzoyl-N-hydroxysulfosuccinimide ester (SMBS). The modifies surfaced react with sulfhydryl groups of unique cysteines mutants of PSI to form layers over solid surfaces. The images of PSI were constructed from x ray crystallographic data published by ${ }^{7}$ PBD 1 JB0. 


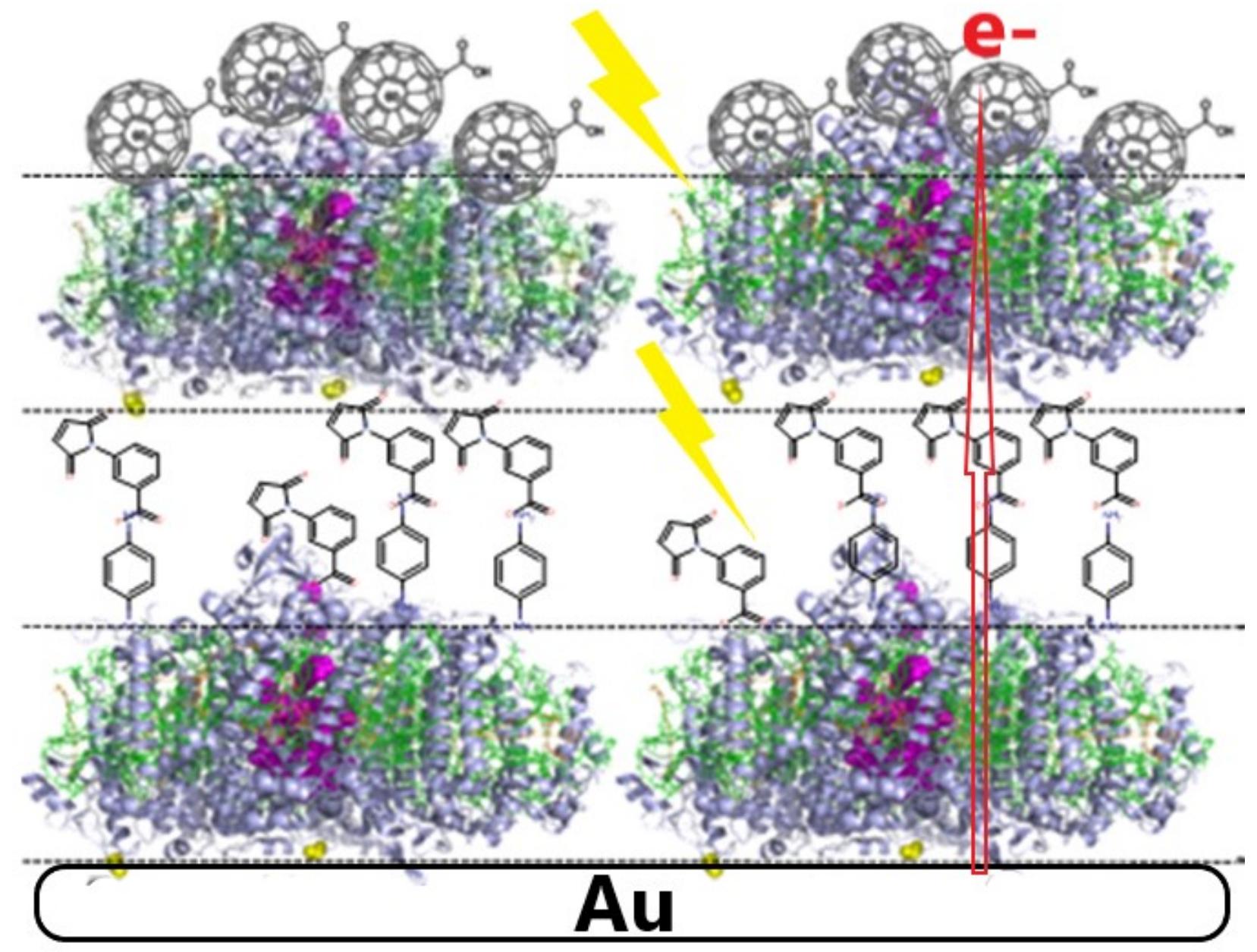

Figure S3. Fabrication of multilayers of PSI. PSI cysteine mutants were self-assembled on Au electrodes. Oriented multilayers were fabricated layer by layer by incubation of a PSI monolayer with the bifuctional linker m-Maleimidobenzoyl-N-hydroxysulfosuccinimide ester (S-MBS), which binds to free amines at the top of the protein layer. The maleimide moiety of the modified layers formed thio-ether bond with the successive layers of PSI. Light induced charge separation drove electrons (red arrow) from the bottom electrode through the covalently bound connecting molecules through the successive layers of PSI. When indicated caboxyfullerens (round images) were added on top of the multilayers. 


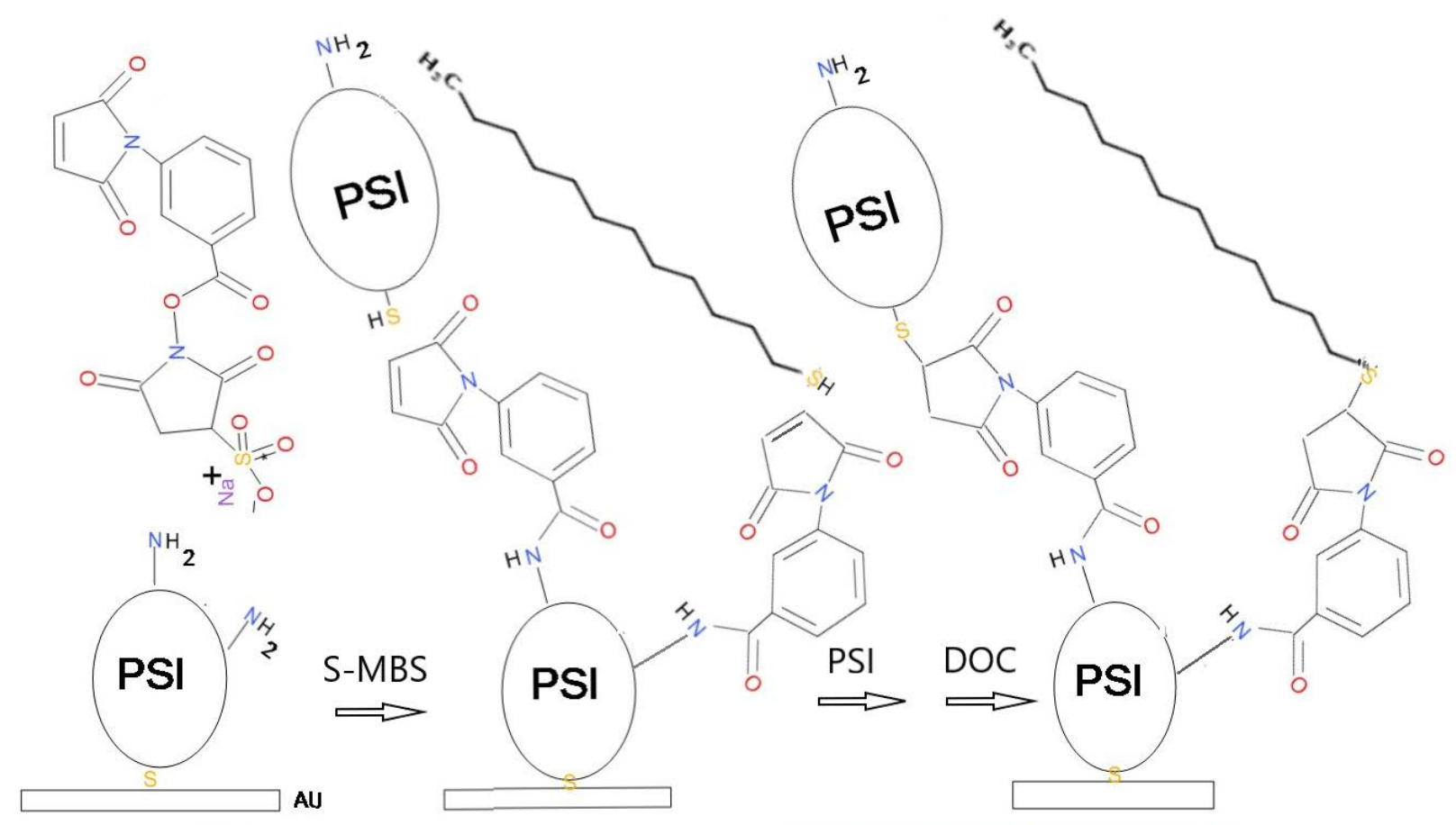

Figure S4. Fabrication of PSI multilayers supplemented with dodecanethiol (DOC). Oriented multilayers were fabricated layer by layer by incubation of a PSI monolayer with the bifuctional linker m-Maleimidobenzoyl-N-hydroxysulfosuccinimide ester (S-MBS), which binds to free amines at the top of the protein layer. The maleimide moiety of the modified layers formed thioether bond with the successive layers of PSI. The free maleimide moieties which did not react with PSI cysteines reacted with the sulfhydrils of DOC. 


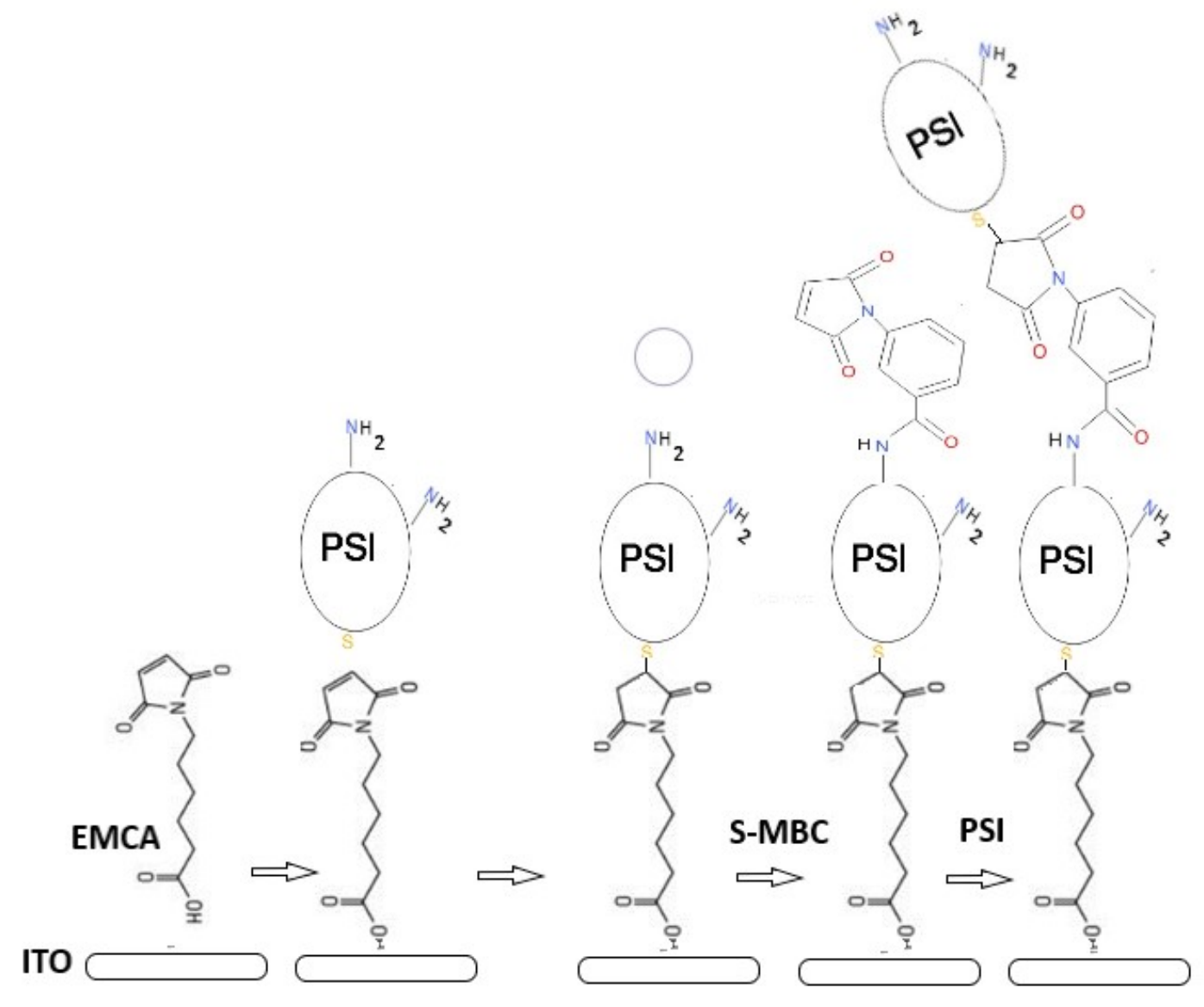

Figure S5. Fabrication of PSI multilayers on ITO surface. The ITO surface is passivated by N- $\varepsilon$ maleimidocaproic acid (EMCA). The carboxyl moiety of EMCA coordinately binds to the metal in the crystal. The maleimide moiety forms a thio-ether bond with the sulfhydryl of the cysteines in PSI. Oriented multilayers were fabricated layer by layer by incubation of a PSI monolayer with the bifuctional linker m-Maleimidobenzoyl-N-hydroxysulfosuccinimide ester (S-MBS), which binds to free amines at the top of the protein layer. The maleimide moiety of the modified layers formed thio-ether bond with the successive layers of PSI.
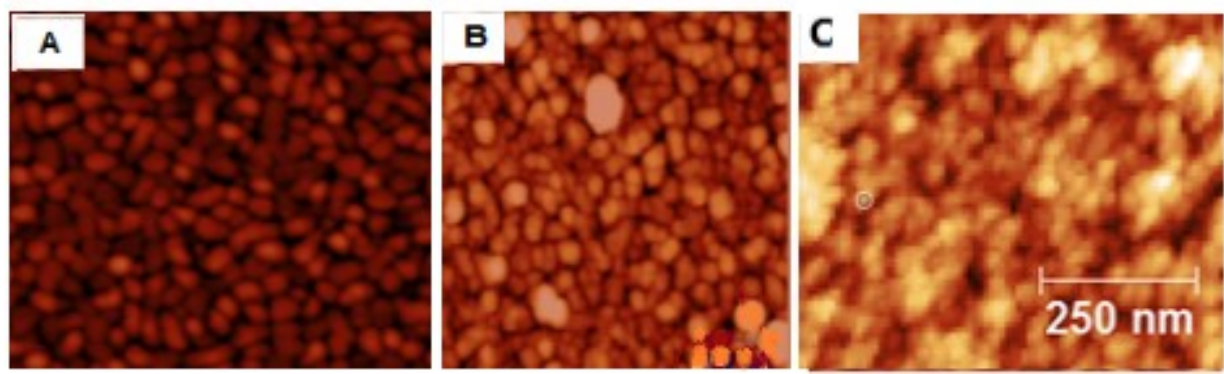
Figure S6. AFM images of PSI multilayers. AFM scans of gold surface (A), single layer (B) and three layers (C) of PSI layers having average height of $1.2 \pm 0.2 \mathrm{~nm}, 9.62 \pm 1.4 \mathrm{~nm}$ and $36.78 \pm 1.6$ $\mathrm{nm}$, respectively. Color code indicate the height of the particles from dark to light brown $0.2 \mathrm{~nm}$ to $40 \mathrm{~nm}$. The size of the particles $\sim 25 \mathrm{~nm}$ corresponds to the size of PSI trimers.

\section{Results Section S}

Spectrophotometry was used to determine the formation of PSI multilayers. The formation of multilayers was determined by measurement. The absorption also increased as a function of the layer number. ${ }^{3}$ The absorption measurements allowed quantitative determination of the fabricated PSI multilayers in the devices. A value of 0.002 A673 per PSI layer was calculated from the absorption spectrum and was in harmony with earlier calculations, assuming coverage of $60 \%$ of the area, $10 \mathrm{~nm}$ light path and $\mathrm{E}_{667} 7.68 \times 10^{4} \mathrm{M}^{-1} \mathrm{~cm}^{-1}{ }^{4}$

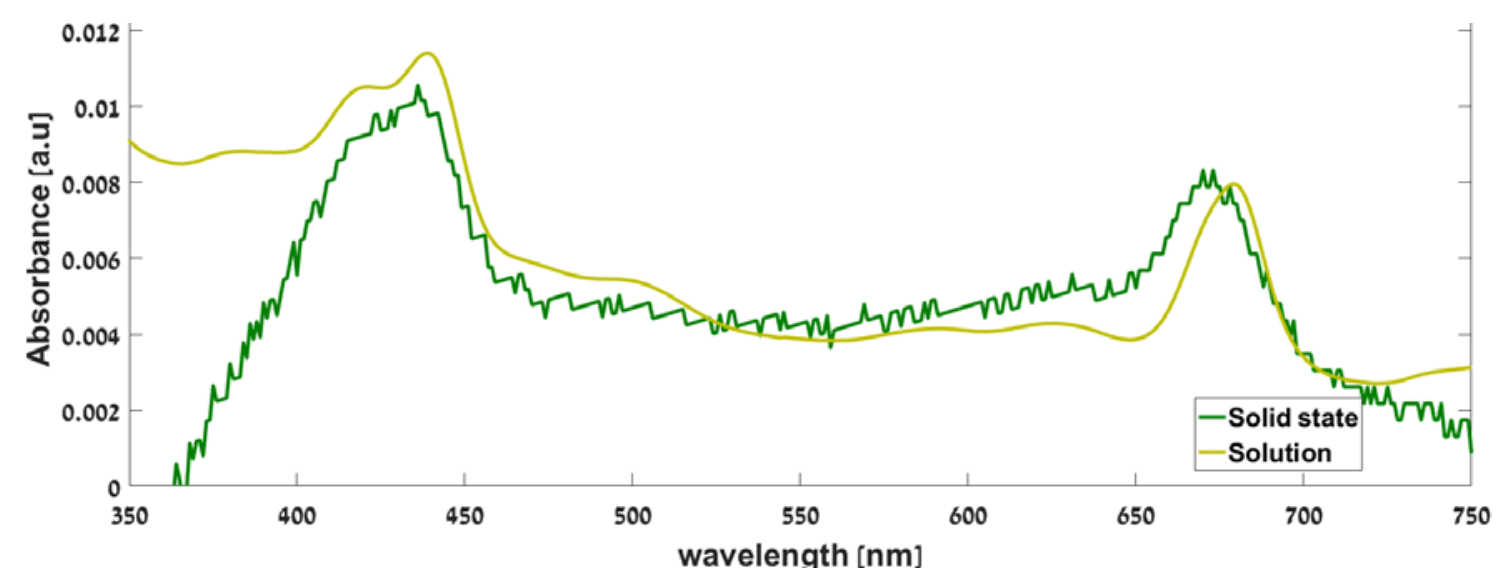

Figure S7. Absorption spectra of PSI. The absorption spectra of three oriented PSI layers on ITO glass (green) compared to the spectrum of PSI in solution (yellow), respectively.

Ellipsometry was used to evaluate the formation of membrane protein layer fabricated on metal surfaces. The height of the layers was measured by a Woollam Alpha-SE Ellipsometer. A value of $8.89 \mathrm{~nm}$ and $19.11 \mathrm{~nm}$ was calculated for a single and double layers of PSI 

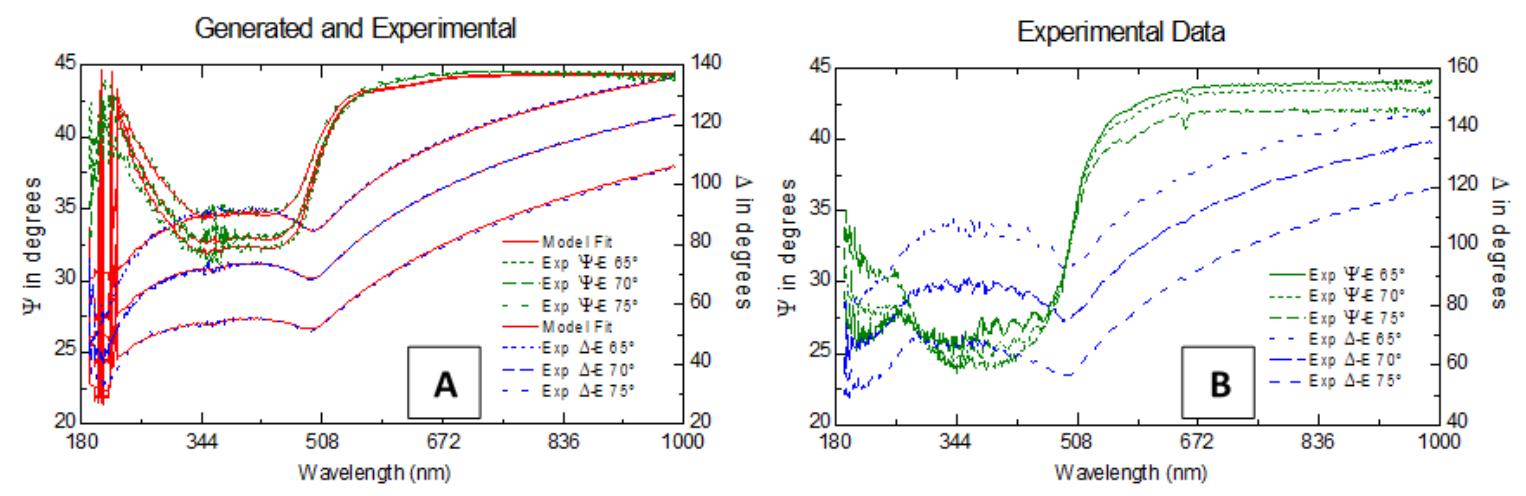

Figure S8. Ellipsometry scans of PSI of layers on gold surface. A. Scans of two layers of PSI. B. Scans of gold surface.

\section{Current voltage measurements:}

As expected from a photovoltaic device both JSC and Voc increased responded to light intensity. As seen in Figure S5 an increase of light at intensities from $0.1 \mathrm{~mW} / \mathrm{cm}^{2}$ to $5 \mathrm{~mW} / \mathrm{cm}^{2}$ resulted in more than a two fold increase in current and voltage.

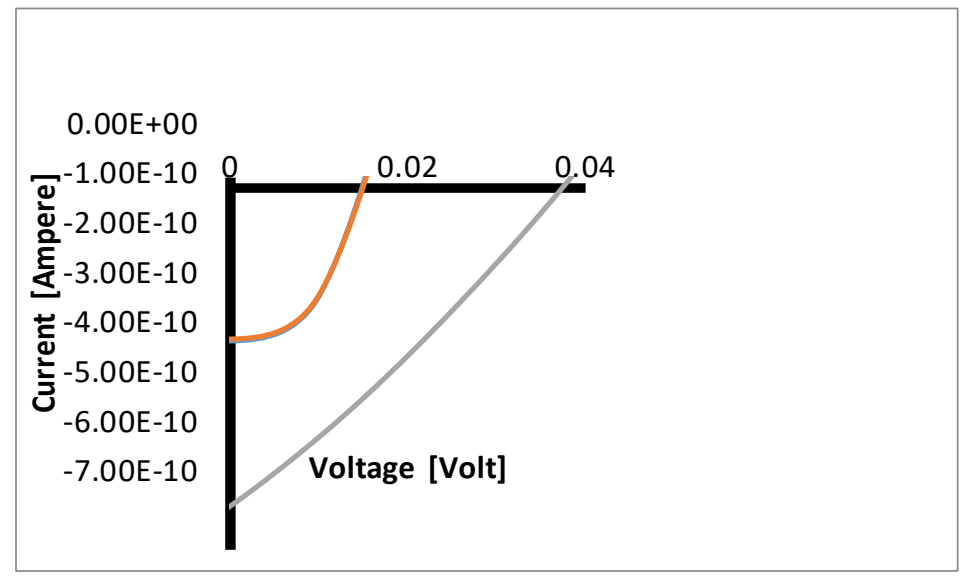

Figure S9. The effect of light intensity on the I/V response of PSI devices. The measurements were of current were conducted on a device consisted of bottom Au and top PEDOT:PSS electrodes and contained 7 oriented PSI layers with PPD bound between the layers. The devices were illuminated by fluoresces light at intensities of $0.1 \mathrm{~mW} / \mathrm{cm}^{2}$ (brown) and $5 \mathrm{~mW} / \mathrm{cm}^{2}$ (gray). 


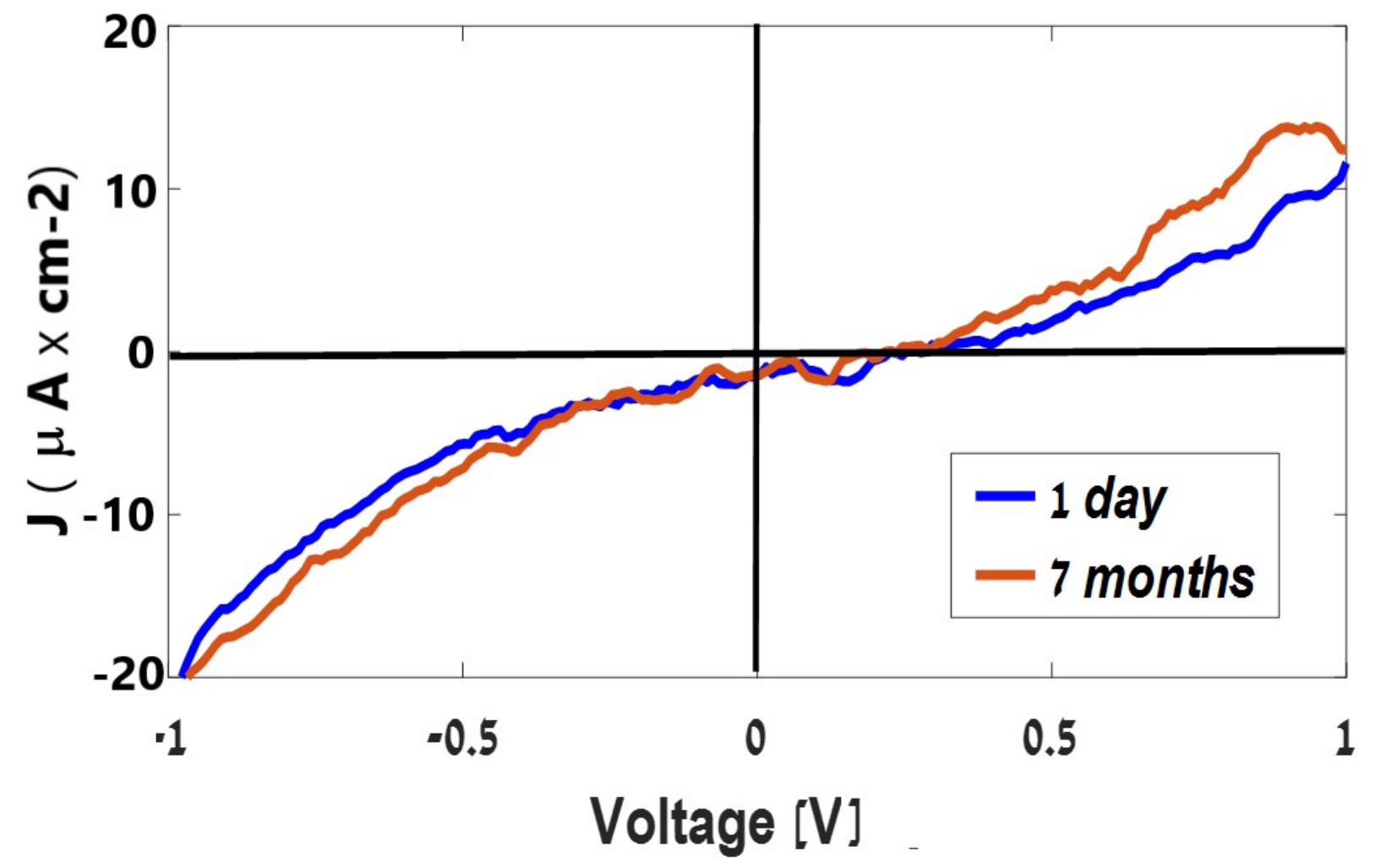

Figure S10. Stability of the cells. Measurements of the same cell after 7 months of storage at room temperature resulted in a small decrease in Voc with but no change in Jsc. The cell yielded Voc of $325.6 \pm 3.00 \mathrm{mV}$ and $312.2 \pm 2.90 \mathrm{mV}$ for day one and seven months, respectively. There was no change in the Jsc of $15.3 \pm 0.09 \mu \mathrm{A}$ after 7 months. The cell contained 7 layers of D31C PSI mutant doped with PPD and DOC, topped with CF on ITO and Au electrodes.

\section{References}

1. Frolov, L.; Rosenwaks, Y.; Carmeli, C.; Carmeli, I., Fabrication of a photoelectronic device by direct chemical binding of the photosynthetic reaction center protein to metal surfaces. Adv. Mater. 2005, 17 (20), 2434-2439.

2. $\quad$ Gong, X. M.; Agalarov, R.; Brettel, K.; Carmeli, C., Control of electron transport in photosystem I by the iron-sulfur cluster $\mathrm{F}-\mathrm{X}$ in response to intra- and intersubunit interactions. $J$. Biol.l Chem. 2003, 278 (21), 19141-19150.

3. Heifler, O.; Carmeli, C.; Carmeli, I., Enhanced Optoelectronics by Oriented Multilayers of Photosystem I Proteins in Dry Hybrid Bio-Solid Devices. J. Phy. Chem. C 2018, 122 (21), 1155011556.

4. $\quad$ Carmeli, I.; Frolov, L.; Carmeli, C.; Richter, S., Photovoltaic activity of photosystem Ibased self-assembled monolayer. J. . Am. Chem. Soc. 2007, 129 (41), 12352-12361.

5. Vatel, O.; Tanimoto, M., Kelvin Probe Force Microscopy for Potential Distribution Measurement of Semiconductor-Devices. Journal. Appl. Phys. 1995, 77 (6), 2358-2362. 
6. Truskett, V. N.; Watts, M. P. C., Trends in imprint lithography for biological applications. Tren. Biotechnol. 2006, 24 (7), 312-317.

7. Jordan, P.; Fromme, P.; Witt, H. T.; Klukas, O.; Saenger, W.; Krauss, N., Three-

dimensional structure of cyanobacterial photosystem I at 2.5 A resolution. Nature 2001, 411 (6840), 909-917. 